\title{
Des Kindes Ernährung - gestern, heute und morgen und vom Überleben zum Besserleben
}

\author{
Nutrition in Infancy - Yesterday, Today and Tomorrow \\ and from the Survival to a Better Life
}

Autor

Institut
W. Nützenadel

Abteilung für Kinder- und Jugendmedizin, Universitätsklinikum Mannheim, Universität Heidelberg
Bibliografie

DOI http://dx.doi.org/ 10.1055/s-0029-1214944

Online-Publikation: 14. 10. 2009

Akt Dermatol 2010; 36:

99-103 @ Georg Thieme

Verlag KG Stuttgart · New York ISSN 0340-2541

Korrespondenzadresse Prof. Dr. Walter Nützenadel Facharzt für Kinderund Jugendmedizin Theodor-Kutzer-Ufer 1-3 68167 Mannheim w.nuetzenadel@web.de

\section{Zusammenfassung $\nabla$}

Die Säuglingsernährung ohne mütterliche Milch wurde bereits im Altertum mit Ammenmilch und auch artfremder Milch durchgeführt, jedoch ermöglichen erst analytische Verfahren zur Zusammensetzung der Milchen Mitte des 19. Jahrhunderts eine Adaptation der Kuhmilch an die Frauenmilch. Zum Ausgleich des hohen Proteinund Mineralgehaltes der Kuhmilch wurde diese verdünnt und zum Ausgleich der durch die Verdünnung verloren gegangenen Kalorien Kohlenhydrate zugefügt. Die richtige Rezeptur für diese Vorgehen war für Jahrzehnte in der Diskussion, bis industriell-technische Verfahren mit der Mo- difikation einzelner Nahrungsbestandteile einen weitgehenden Angleich der chemischen Komposition der Kuhmilch an die Frauenmilch ermöglichten. Neben den Veränderungen der Zusammensetzung haben andere Innovationen, wie hygienische Standards, die Entwicklung von Saugern und Flaschen, von Pulvermilchen u.a., zu den Erfolgen der künstlichen Ernährung beigetragen. Die heute industriell hergestellten Säuglingsnahrungen sind in ihrer Zusammensetzung weitgehend standardisiert und haben gegenüber der Muttermilch nur noch geringe nutritive Nachteile; wegen zahlreicher biologischer und psychologischer Vorteile ist Stillen jedoch die bevorzugte Art der Säuglingsernährung.

\section{Einleitung}

$\nabla$

„Des Kindes Ernährung“ ist der Titel eines berühmten Buches von Adalbert Czerny, einem der Pioniere der Pädiatrie; den Inhalt des Buches und die Reputation des Autors feierte 1932 eine Festschrift des Journals of Pediatrics folgendermaßen: „this work is a bible“ und „at a time when pediatrics in America was still in its swaddling clothes, German masters took us into their clinics as students and by their inspiration and training played a part in the development of pediatrics in America, of which they may feel proud“ [1].

Dieser Rückblick in eine glanzvolle Zeit der deutschen Pädiatrie zeigt die Bedeutung der Säuglingsernährung innerhalb des Faches und ist somit auch eine passende Einleitung zu den nachfolgenden historischen Betrachtungen. In dieser Darstellung möchte ich einen Bogen spannen von den Problemen des „Überlebens“ der Kinder vor mehr als 100 Jahren zu heute möglichen Verbesserungen der frühkindlichen Ernährung mit einem „Besserleben“ auch jenseits der Kindheit.

\section{Anfänge der künstlichen Säuglingsernährung \\ $\nabla$}

Den Zeitpunkt des Beginns einer künstlichen Ernährung festzulegen, ist nicht einfach, jedoch scheint bereits im Altertum eine Ernährung von Säuglingen ohne mütterliches Stillen mit Ammenmilch und auch artfremder Milch möglich gewesen zu sein. Die Ernährung mit artfremder Milch hatte zwei Voraussetzungen: die Tierhaltung und ein Füttern ohne Anlegen an die Brust. Grabbeigaben von Tongefäßen mit einer Formgebung, die das Saugen eines Kindes erlaubt, sind bereits aus dem griechisch-römischen Altertum bekannt, und die Skulptur der Romulus und Remus säugenden Wölfin in Rom zeugt von der Vorstellbarkeit einer Tiermilchernährung zu dieser Zeit ( $\bullet$ Abb. 1). Eine dauerhaft erfolgreiche Ernährung mit Tiermilch war jedoch unter den gegebenen Verhältnissen sicher eher die Ausnahme, während Ammenmilch bereits als gute Alternative zur Muttermilch genutzt wurde.

Vom Altertum bis zum Übergang in die Neuzeit verharrte die Medizin weitgehend in den Vorstellungen, die bereits im Altertum galten; erst mit 
dem Erwachen einer naturwissenschaftlich ausgerichteten Wissenschaft und dem wachsenden Interesse an Anatomie und Medizin nach 1450 erscheinen medizinische Publikationen und Bücher, darunter auch solche mit einem pädiatrischen Fokus. Die künstliche Ernährung findet darin keine Erwähnung, sieht man von Bemerkungen zur moralischen und gesundheitlichen Verfassung von Ammen ab.

Als eigenständiges Fach beginnt die Kinderheilkunde etwa in der Mitte des 19. Jahrhunderts zu existieren. Dies ist begleitet von einer rasanten Zunahme naturwissenschaftlicher Erkenntnisse und vollzieht sich in einer Epoche der Entwicklung anderer medizinischer Fächer zugleich mit neuen technischen Methoden und Verfahren. Gleichzeitig ist diese Epoche geprägt von der industriellen Revolution des 19. Jahrhunderts mit ihrem Wandel sozialer und gesellschaftlicher Strukturen. Für die Pädiatrie lässt sich das Tempo und auch die Art der Veränderungen an Folgendem ablesen: Zwischen 1840-1850 werden 9 Kinderkliniken in Deutschland gegründet, 50 Jahre später finden wir eine hohe zweistellige Zahl; die erste deutschsprachige pädiatrische Zeitschrift, das Journal für Kinderheilkunde, erscheint 1842, um 1900 zählte man bereits mehr als 5 deutschsprachige pädiatrische Zeitschriften; 1877 erscheint das erste Handbuch der Kinderkrankheiten von C. Gerhardt; und 1883 wird mit 97 Mitgliedern die Gesellschaft für Kinderheilkunde gegründet.

Welche Probleme stellten sich dem neuen Fach? Die Sterblichkeit im ersten Lebensjahr war mit 20 bis 30\% erschreckend hoch; die dafür verantwortlichen Faktoren waren vielfältig, unter ihnen war die Art der Ernährung ohne stillende Mutter besonders bedeutsam. So führten eine unzureichende Ernährung wie auch Infektionskrankheiten, besonders in den Kliniken, zu katastrophal schlechten Ergebnissen bei der stationären Behandlungen der Kinder. Von 1874 bis 1884 lag die Letalität in der Kinderklinik der Charité bei $78 \%$ und nichts charakterisiert die bestehende Hilflosigkeit besser als die Empfehlung des Direktors der Klinik Henoch an seinen Nachfolger Heubner, die Säuglingsabteilung zu schließen. Die damals vorhandenen Möglichkeiten der künstlichen Ernährung waren beschränkt auf Mischungen aus Getreide mit und ohne Fleischzusatz sowie auf die Verwendung nativer Tiermilch. Die praktische Nutzung und die hygienischen Risiken von Tiermilch demonstriert die Abb. 2. Eine der ersten Milchmodifikationen für die Säuglingsernährung war die „Suppe für Säuglinge“ von Justus Liebig. Die einleitenden Worte in seiner Publikation umreißen die sich stellende Aufgabe einer künstlichen Säuglingsernährung: „es ist leicht verständlich, dass ein Kind, welchem die Milch seiner Mutter versagt ist, ohne Amme nur dann in der rechten Weise ernährt werden kann, wenn die Speise, die man ihm reicht, denselben Ernährungswert wie die Frauenmilch hat" [2].

Seine Überlegungen zum Protein- und Energiestoffwechsel und deren Besonderheiten während des Wachstums waren neu und basierten auf Analysen, Beobachtungen und physiologischen Überlegungen und weniger auf tradierten Festlegungen und Vorstellungen. Damit zeigten sich neue Denkansätzen sowie die Einbeziehung der Erkenntnisse anderer wissenschaftlicher Disziplinen. Die Untersuchungen der Kuh- und Muttermilch ergaben einen hohen Anteil an Kasein und Mineralstoffen in der Kuhmilch, und mit dem von Philipp Biedert, der bei Liebig in Gießen promovierte, empfohlenen „Ramogen“ begann eine lang anhaltende Diskussion um „den besten Ausgleich der chemischen Verschiedenheiten zwischen Frauen- und Kuhmilch“, wie Heubner es formuliert hat [3].

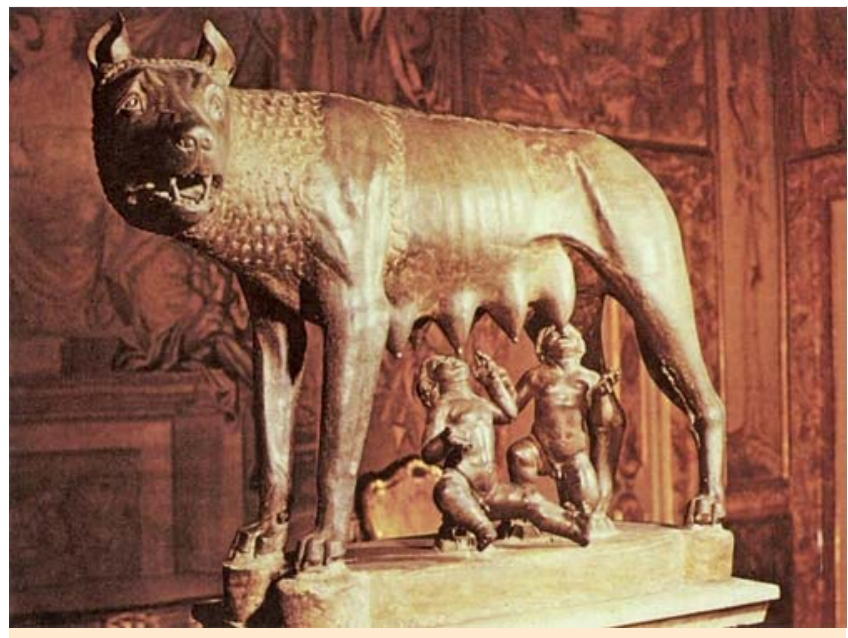

Abb. 1 Säuglingsernährung durch Tiermilch. Skulptur der Romulus und Remus säugenden Wölfin, Palazzo del Conversatori, Rom.

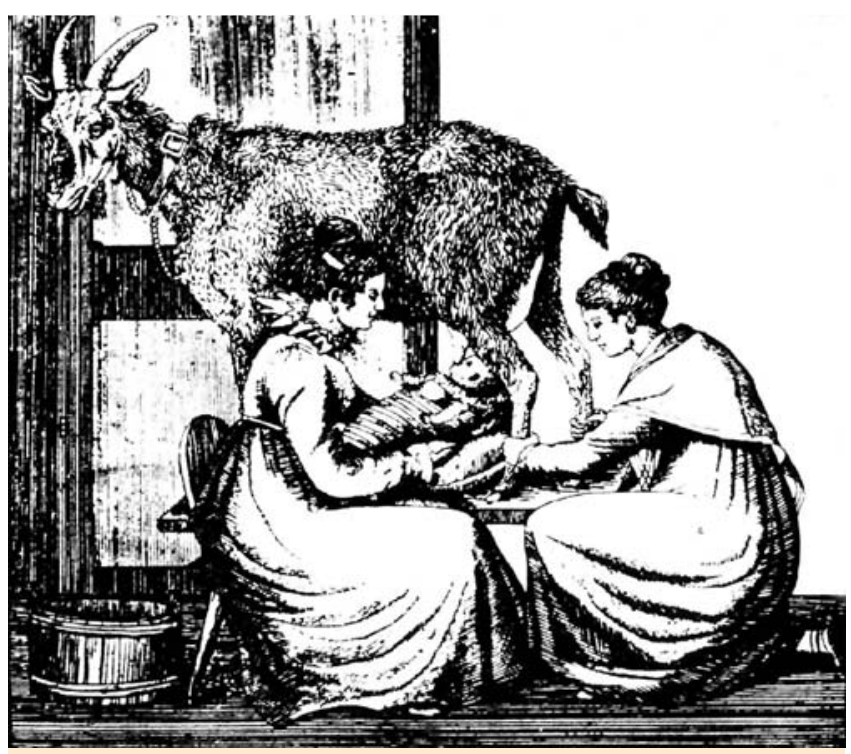

Abb. 2 Künstliche Ernährung mit Tiermilch (Bild: aus einer Klinik in Frankreich).

Die Analysen der Kuh- und Frauenmilch legten die Verdünnung der Kuhmilch zur Reduktion des Proteinanteiles sowie die Zugabe von Kohlenhydraten zur Kalorienanreicherung als wichtige Schritte zu einer verbesserten Verträglichkeit der Kuhmilchernährung nahe. Das Was und Wie möglicher Modifikationen war von da an für Jahrzehnte Gegenstand vieler Publikationen, und nicht alle Vorschläge führten zu einer verbesserten Ernährung des Säuglings. Dennoch zeigten sich mit der Zeit Erfolge der künstlichen Ernährung, wenngleich andere Veränderungen, die nicht die Zusammensetzung der Nahrung betrafen, dazu wesentlich beigetragen haben. Besonders bedeutsam dafür waren die neuen Erkenntnisse zur Hygiene und Bakteriologie. Durch die Arbeiten von Semmelweiß, Koch, Pasteur, Gram, von Behring und anderen wurde in der zweiten Hälfte des 19. Jahrhunderts die bakteriologische Ätiologie für zahlreiche Erkrankungen bewiesen. Milch erwies sich als gutes Nährmedium für Bakterien und die bakterielle Kontamination der Säuglingsnahrungen verschlechterte die Verträglichkeit der Kuhmilchernährung erheblich [4]. 
Entsprechend gewannen hygienische Maßnahmen bei der Milchgewinnung und -verarbeitung sowie die Sterilisation der Nahrungen vor dem Füttern schon vor der Jahrhundertwende große Bedeutung.

\section{Ernährungsstörungen und Toxikose}

Mit den Begriffen „Ernährungsstörungen“ und „Toxikose“ schufen Czerny, Keller und Finkelstein zu Beginn des 19. Jahrhunderts ein damals neues und als revolutionär empfundenes Konzept, das Ernährung und Krankheit des Kindes in einen besonderen ätiologischen Zusammenhang stellt. In dem Buch „Des Kindes Ernährung und Ernährungsstörungen und Ernährungstherapie“ von Czerny sowie der Publikation von Finkelstein „Über alimentäre Intoxikation im Säuglingsalter“ wurden die Art der Ernährung und die Pathogenese der Diarrhö kausal verbunden [5,6].

Das Konzept der beiden Autoren war damals überzeugend, fand weltweite Anerkennung und begründete die bereits erwähnte Reputation der Autoren. Wenige Worte aus der Arbeit von Finkelstein offenbaren die unterstellte Kindsgefährdung aus der Ernährung: „wir sind befugt, den Symptomkomplex der Intoxikation als Ausdruck einer alimentären Vergiftung... anzusehen, die Intoxikationssymptome im Verlauf von Ernährungsstörungen sind alimentärer Natur, sie entstehen, wenn gewisse Nahrungsbestandteile in größerer Menge zugeführt werden“. Woraus leitete sich die Annahme einer möglichen Vergiftung durch die Ernährung ab? Bereits bei der Suche nach den Ursachen der schlechten Verträglichkeit der Kuhmilch wurden toxische Effekte einzelner Bestandteile der Kuhmilch vermutet. Biedert unterstellte dem Kasein eine ungünstige Wirkung, und so war die Annahme ungünstiger Effekte der Nahrung oder ihrer Bestandteile bereits vor dem Toxikosekonzept der drei Autoren in der Diskussion. Da andererseits Kinder auch mit Kuhmilchzubereitungen gediehen, unterstellten die Autoren eine eingeschränkte Verträglichkeit für Nährstoffe im unreifen Darm des Säuglings. Danach führt eine qualitativ oder auch quantitativ falsche Ernährung in Verbindung mit anderen exogenen oder endogenen Faktoren zum schrittweisen Überschreiten der Verträglichkeit und dem Auftreten von Symptomen. Klinisch wurde eine sich schrittweise entwickelnde Symptomatik angenommen, deren Stufen als alimentäre Dekompensation, Dyspepsie und allgemeine Dekompensation bezeichnet wurden. Die vermuteten Toxine sollten im Darm oder auch endogen durch das Überschreiten der Toleranz gebildet werden. Die danach einsetzende Forschung auf der Suche nach diesen Toxinen war vergeblich, dagegen war die Kandidatenliste der vermuteten Toxine, wie Peptone, Phenole, Thiole, Skatol, Indol und Histamin sowie Serotonin, Hydroxyfettsäuren und anderen Substanzen, recht umfangreich. Die unterstellte Kindsgefährdung durch die Ernährung intensivierte die bereits bestehende Debatte über eine richtige Ernährung, führte zu Empfehlungen einer sehr vorsichtigen Nahrungszufuhr und resultierte in einer Vielfalt von Ernährungsvorschlägen. Diese hatten oft keine rationale Begründung und fußten eher auf den persönlichen Erfahrungen der Autoren. Eine Vorstellung der Gestaltungslust vermittelt die - Abb. 3 aus dem Handbuch für Kinderheilkunde von 1930. Basis der Vorschläge war die „Verdünnung der Kuhmilch mit Schleim bzw. Mehlabkochung unter Zusatz von Zucker“.

Der Verdünnungsgrad und die Art der zugesetzten Kohlenhydrate waren Gegenstand umfangreicher Untersuchungen, und es ergaben sich eine Fülle von Vorschlägen über die Art der Ernährung und wie bei Nichtgedeihen vorzugehen sei [7].

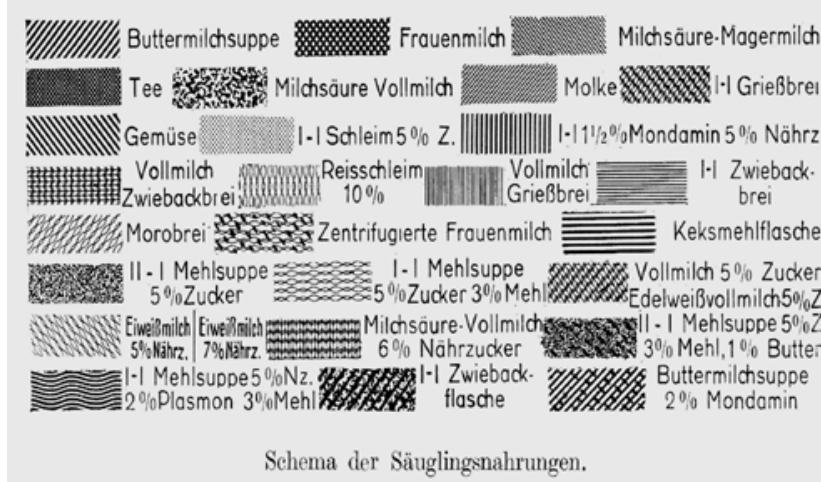

Abb. 3 Vielfalt der Säuglingsnahrungen mit grafischer Darstellung einzelner Nahrungen für das Kurvenblatt aus dem „Handbuch für Kinderkrankheiten“ von Schlossmann (1930).

\section{Wahre Ernährungsstörungen \\ $\nabla$}

Das Konzept von Czerny und Finkelstein war zu seiner Zeit überzeugend und hat die Gestaltung der kindlichen Ernährung sowie die Behandlung ihrer Störungen lange stark beeinflusst. Es unterschätzte die Bedeutung der Dehydratation bei der Diarrhö, führte zu einer oft unzureichenden Nährstoffzufuhr und hat nachfolgend einer wissenschaftlichen Überprüfung nicht standgehalten. Dennoch existiert die Kausalität einer Pathogenese „ex alimentatione“ für bestimmte Erkrankungen auch heute, und einige davon wurden bereits am Anfang des 20. Jahrhunderts publiziert. Heubner beschreibt 1909, ein Jahr nach der Publikation von Herter, die Symptome der Zöliakie, ohne diese Bezeichnung zu nutzen und auch ohne Hinweis auf die Publikation von Samuel Gee aus dem Jahr 1888 mit dem Titel „On the coeliac affection“. Die Zöliakie wurde im deutschen Schrifttum lange als Herter-Heubner-Erkrankung bezeichnet, ehe sich die Diagnose Zöliakie dafür durchsetzte. Während Herter mit dem Titel seiner Arbeit „Monograph on infantilism from chronic intestinal infection“ der Infektion eine besondere Rolle zuwies, betonte Heubner mit seinem Titel „Über schwere Verdauungsinsuffiziens beim Kinde jenseits des Säuglingsalters“ die für die kindliche Symptomatik so bedeutsame Malabsorption [8,9].

Erfolgte die Aufdeckung der nahrungsbedingten Ätiologie der Zöliakie erst Jahrzehnte später, so hat Schlossmann in Dresden bereits 1905 eine Arbeit mit dem Titel „Über die Giftwirkung des artfremden Eiweißes in der Milch auf den Organismus des Säuglings“ publiziert [10].

Nicht ganz deutlich wird, welche Symptome mit dieser Giftwirkung gemeint waren. Mit der Erwähnung von Antikörpern in der Arbeit lässt sich aber eine Brücke zur Allergie schlagen, ein Begriff, der erst 1906 von v. Pirque geprägt wurde. Die typischen Symptome einer allergischen Reaktion auf Kuhmilch lassen sich aber in einer Arbeit von Freund mit dem Titel „Über Idiosynkrasie gegenüber Kuhmilch bei Säuglingen“ aus dem Jahr 1909 erkennen: 4 Monate nach dem Abstillen traten bei Kuhmilchfütterung Erbrechen, Durchfall und Kollaps auf. Aus den Ergebnissen verschiedener Provokationstests wurde die Schlussfolgerung einer vorliegenden Idiosynkrasie gegen Kuhmilch gezogen [11].

Unter dem Begriff „Idiosynkrasie gegen Kuhmilch“ finden sich dann im deutschen Schrifttum bis 1914 vier weitere Arbeiten. Der amerikanische Pädiater Ruhräh beschrieb 1911 die Unverträglichkeit für Sojaprotein, und sein Landsmann Schloss beschreibt 1912 eine Urtikaria nach dem Genuss von Eiern, Man- 
deln und Hafer bei einem 8-jährigen Jungen. Mit diesen Publikationen ist die Nahrungsmittelallergie bereits Anfang des 20. Jahrhunderts klinisch gut charakterisiert. Erstaunlich ist, dass diese Diagnose danach kaum gestellt wurde; erst nach 1960 etablieren sich Nahrungsmittelallergien als eigenständige Diagnosen in der Literatur. Man kann vermuten, dass zwischenzeitlich adverse Reaktionen nach der Nahrungsaufnahme als toxische Effekte durch ein Überschreiten der Toleranz im Sinne des Konzeptes von Czerny gedeutet wurden.

\section{Von der „Rezeptur“ der Säuglingsnahrungen zur industriell hergestellter Flaschennahrung $\nabla$}

Die dargestellte Vielfalt bei der Zubereitung von Flaschennahrungen erforderte eine apotheken-genaue Herstellung nach Rezept. Die dafür notwendige Logistik wurde durch die Einrichtung von Milchküchen in den Kliniken garantiert. Auch außerhalb von Kliniken wurden mancherorts Strukturen mit einer zentralen $\mathrm{Zu}-$ bereitung von Säuglingsmilchen und deren Abgabe an Mütter geschaffen. Neben der einwandfreien Zusammensetzung der Nahrungen sicherte die Herstellung in den Milchküchen auch gute hygienische Bedingungen, etwa durch die Flaschensterilisation und durch die Sicherheit beim Säubern der Flaschen und Sauger ( Abb. 4). Die Bedeutung der Milchküche zeigte sich darin, dass die Weiterbildung pädiatrischer Assistenten ein Praktikum in der Milchküche einschloss. Die Entwicklung der künstlichen Säuglingsernährung wird sehr früh begleitet von der Herstellung industrieller Produkte für die Ernährung des Kindes. So führt bereits 1877 Jacobi im Handbuch der Kinderkrankheiten unter Surrogaten folgende auf: Kindermehle von Nestle, Gerber, Faust und Schuster; und in der ersten Hälfte des 20. Jahrhunderts waren industriell hergestellte Präparate mit Kohlenhydraten zur Kalorienanreicherung der verdünnten Kuhmilch weit verbreitet [12]. Die Erfolge der künstlichen Ernährung waren überdies auch von technischen und industriellen Innovationen abhängig, die nicht die Zusammensetzung der Säuglingsnahrung betrafen. Zu nennen wären die Sauger - erst aus Gummi, später aus Kunststoff -, hitzebeständige Glas- und Kunststoffflaschen und die Möglichkeit der Pulvermilchherstellung. Die Zubereitung der Säuglingsnahrung im Haushalt oder in Milchküchen mit Verdünnen der Kuhmilch und Anreicherung mit Kohlenhydraten in Form der Halb- und Zweidrittelmilch wurde ab der Mitte des 20. Jahrhunderts weitgehend abgelöst durch industriell hergestellte Fertignahrungen, meist in Pulverform. Den vielen Vorteilen (Hygiene, verfeinerte Anpassung an die Muttermilch, einfache Handhabung, Konstanz der Zusammensetzung u.a.) stehen höhere Kosten gegenüber. Insgesamt konnte aber mit neuen technischen Verfahren der Grad der Humanisierung in einem Maße gesteigert werden, die das einfache Verdünnen der Kuhmilch und ein nachfolgendes Anreichern mit Kohlenhydraten nicht erlaubte. Einzelne Nahrungsmittelbestandteile, wie Eiweiß oder Fette, wurden spezifisch modifiziert, und der Gehalt an Vitaminen, Mineralien und anderen Spurenelementen in den Nahrungen konnte altersgerecht eingestellt werden. Standards für die Säuglingsnahrungen wurden fixiert und kodifiziert und damit eine hohe Sicherheit der kommerziell vertriebenen Säuglingsnahrungen geschaffen. Folgemilchen wurden für die Ernährung in der zweiten Hälfte des ersten Lebensjahres entwickelt. Ihre Komposition ist weniger eng an der Muttermilch ausgerichtet, aber die Verwendung komplexer Kohlenhydrate ermöglicht einen höheren Sättigungsgrad. Trotz der erreichten Adaptation an die Muttermilch werden

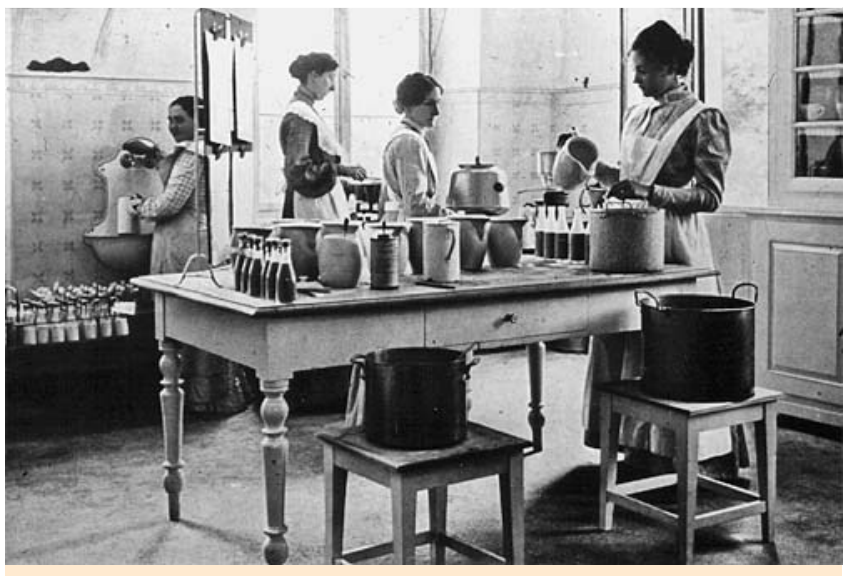

Abb. 4 Nahrungszubereitung in der Milchküche der Kinderklinik Heidelberg etwa um 1930.

weitere Verbesserungen etwa bei der Optimierung der Zufuhr an ungesättigten Fettsäuren oder mit dem Zusatz von Präbiotika angestrebt. Insgesamt ist aber die Adaptation an die Muttermilch weit fortgeschritten, und somit ist das nutritive Risiko einer künstliche Säuglingsernährung klein geworden. Morbiditätsund Mortalitätsunterschiede bei Mutter- oder Flaschenmilchernährung sind deshalb heute nur noch gering.

\section{Langzeiteffekte der Säuglingsernährung \\ $\nabla$}

In dieser historischen Betrachtung sollte ein Bogen von der Vergangenheit mit einem besseren „Überleben“ der Kinder zu Gegenwart und Zukunft mit einem „Besserleben“ durch Modifikationen der Kinderernährung gespannt werden. Die Betrachtung der Säuglingsernährung allein unter dem Gesichtspunkt „gedeihender Säugling“ greift heute zu kurz. Es zeigt sich nämlich, dass die Art der frühkindlichen Ernährung nicht nur eine Bedeutung für das Gedeihen des Säugling hat. Der Begriff „Programming“ umfasst ein Konzept, bei dem ein Stimulus oder Insult während einer sensiblen Periode in der Entwicklung biologische Effekte im späteren Leben auslöst. Solche Effekte sind für die frühkindliche Ernährung im Tierexperiment nachweisbar. Für den Menschen konnten Veränderungen der Inzidenzraten für den Diabetes Typ 1 und 2, für die Adipositas, für die Hypertonie und für andere Erkrankungen im Erwachsenenalter durch Modifikationen der Säuglingsernährung gezeigt werden [13].

Diese Langzeiteffekte sind überraschend und in ihren Mechanismen nicht einfach zu verstehen. Jedoch ist lange bekannt, dass eine unzureichende intra-uterine Ernährung mit einer im späteren Leben auftretenden Morbidität verbunden sein kann. Beispiele sind eine unzureichende Vitamin-B12-Versorgung bei veganer Ernährung der Mütter oder der präventive Effekt einer Folatsubstitution für die Inzidenzrate der Spina Bifida. Art der Säuglingsernährung und ihr Einfluss auf Erkrankungen im Erwachsenenalter sind neue Felder der Forschung zur frühkindlichen Ernährung. Weitere Optimierungen der Ernährung des Säuglings eröffnen somit neben dem weitgehend gesicherten „Überleben“ auch Chancen für ein „Besserleben“ in späteren Lebensjahren. 


\section{Abstract}

\section{Nutrition in Infancy - Yesterday, Today and Tomorrow and from the Survival to a Better Life \\ $\nabla$}

Infant's nutrition by wet nurses or with milk of animals has been reported from ancient times. Successful artificial feeding and the adaptation process of humanization of cow's milk started with the chemical analysis of milks in the midst of the 19. century. The high contents of proteins and minerals were corrected by dilution and adding carbohydrates to restore the density of calories. The discussion about the details of the best composition of the formula lasted several decades until advanced technical procedures allowed a very similar chemical composition of human and artificial milk; other technical innovation, like improved hygiene, development of feeding-bottles and dummies, of powdermilk and other, contributed to the success of artificial feeding. Now, commercially available infant's formula are standardized, their chemical composition is quite similar to mother's milk, and the nutritional disadvantages of artificial feeding compared to breast feeding is quite small; but biological and psychological factors make mother's milk the best choice for infant feeding.

\section{Literatur}

1 Helmholtz HF. Festschrift Adalbert Czerny - An Appreciation. J Pediatr. 1933; 3: 5-6

2 von LiebigJ. Suppe für Säuglinge. Braunschweig: Friedrich Vieweg und Sohn, 1866

3 Biedert $P$. Untersuchungen über die chemischen Unterschiede der Menschen- und Kuhmilch. [Dissertation]. Gießen: 1869

4 Ogie JW. Die Milch und das Mikroskop. Jhrb Kinderheilk 1874; 7: 260 264

5 Czerny A, Keller A. Des Kindes Ernährung und Ernährungsstörungen und Ernährungstherapie. Leipzig: Franz Deuticke, 1913

6 Finkelstein $H$. Über die alimentäre Intoxikation im Säuglingsalter. Jhrb Kinderheilk 1908; 65: 1: 263-269

7 Lust F. Diagnostik und Therapie der Kinderkrankheiten. 6 Aufl. Berlin: Urban u. Schwarzenberg, 1929: 22

8 Herter CA. Intestinaler Infantilismus. Jhrb Kinderheilk 1909; 69: 722 729

9 Heubner 0 . Über die schwere Verdauungsinsuffiziens beim Kinde jenseits des Säuglingsalters. Jhrb Kinderheilk 1909; 70: 667-671

10 Schlossmann A. Über die Giftwirkung des artfremden Eiweißes in der Milch auf den Organismus des Säuglings. Arch Kinderheilk 1905; 41: 99- 104

11 Freund $A$. Über Idiosynkrasie gegenüber Kuhmilch bei Säuglingen. Arch Kinderheilk 1909; 51: 385-394

12 Jacobi A. Die Plege und Ernährung des Kindes - Die gebräuchlichsten Surrogate. In: Gerhardt C (Hrsg). Handbuch der Kinderkrankheiten. Tübingen: Lauppsche Buchhandlung, 1877: 397

13 Lucas $A$. The developmental origins of adult health and well-being. In: Koletzko B, Dodds P, Akerbloom H, Ashwell M (Hrsg). Early nutrition and its later consequences. Heidelberg: Springer, 2005 